\title{
Correction to: Information Security Practice and Experience
}

\author{
Robert Deng (1), Feng Bao, Guilin Wang, Jian Shen, Mark Ryan, \\ Weizhi Meng, and Ding Wang
}

\section{Correction to: \\ R. Deng et al. (Eds.): Information Security Practice and Experience, LNCS 13107, https://doi.org/10.1007/978-3-030-93206-0}

The original version of this chapter "Out of Non-linearity: Search Impossible Differentials by the Bitwise Characteristic Matrix" contained the following errors which have been now corrected:

(1) In section 2.2, a missing closing parenthesis has been included.

(2) In section 3.1, a sentence was missing.

(3) In section 3.2, an extra dot has been removed from first display equation.

(4) In appendix A, the first 3 matrices which has been written in $\{\backslash$ scriptsize $\}$, due to the changes of the pages, we have changed the $\{\backslash$ scriptsize $\}$ to $\{\backslash$ small $\}$ for the first 3 matrices, and the other remain the same.

The original version of this chapter "Automatic Key Recovery of Feistel Ciphers: Application to SIMON and SIMECK" contained the following error which has been now corrected:

In the introduction part, "Crypto 2020" should be "Eurocrypt 2020".

The updated version of these chapters can be found at https://doi.org/10.1007/978-3-030-93206-0_6

https://doi.org/10.1007/978-3-030-93206-0_10 